\title{
CORRIGENDUM
}

\section{Impact of vitamin D supplementation on adiposity in African-Americans}

PD Chandler, JB Scott, BF Drake, K Ng, AT Chan, BW Hollis, KM Emmons, EL Giovannucci, CS Fuchs and GG Bennett

Nutrition \& Diabetes (2015) 5, e164; doi:10.1038/nutd.2015.14; published online 15 June 2015

Correction to: Nutrition \& Diabetes (2015) 5, e147; doi:10.1038/ nutd.2014.44; Published online 19 January 2015

The authors wish to make readers aware that the wrong units were used in Table 2 . Weight at 6 months was incorrectly reported in pounds instead of kilograms at 6 months, as was change in weight by months.

The amended table is presented below.

In the text baseline weight estimate was incorrectly reported in pounds instead of kilograms.

The following sub sections from the Results section have been amended:

Association of baseline 25(OH)D and $\mathrm{BMI}$ and weight

Baseline weight and baseline BMI were not significant predictors of baseline 25(OH)D (estimate (s.e.), $P$-value: $\mathrm{BMI},-0.04(0.07)$, $P=0.55$; weight, $-0.04(0.02), P=0.06)$. Baseline $25(\mathrm{OH}) \mathrm{D}$ was not a significant predictor of baseline $\mathrm{BMI}$ or baseline weight (BMI, -0.03 (0.05), $P=0.55$, weight, $-0.26(0.13), P=0.06)$.

Impact of vitamin D supplementation on BMI and weight

The primary efficacy analyses of vitamin D3 supplementation on weight and BMI are shown in Table 2. In linear regression with the dose of vitamin D3 (per $1000 \mathrm{IU}$ per day) as the independent variable and the 3-month change in weight (or 3-month change in $\mathrm{BMI}$ ) as the dependent variable, no effect of vitamin D3 on change in BMI or weight was observed. For each additional $1000 \mathrm{IU}$ per day of vitamin D3, BMI increased by estimate (s.e.)): 0.01 (0.039); $P=0.78$ and weight increased by estimate (SE):0.03 (0.11); $P=0.81$. At 6 months, no significant change in $\mathrm{BMI}$ or weight was observed (Table 2 ).

The basic message of the paper is not altered. There is no effect of vitamin D supplementation on weight or BMI.

The authors apologise for any inconvenience caused. 


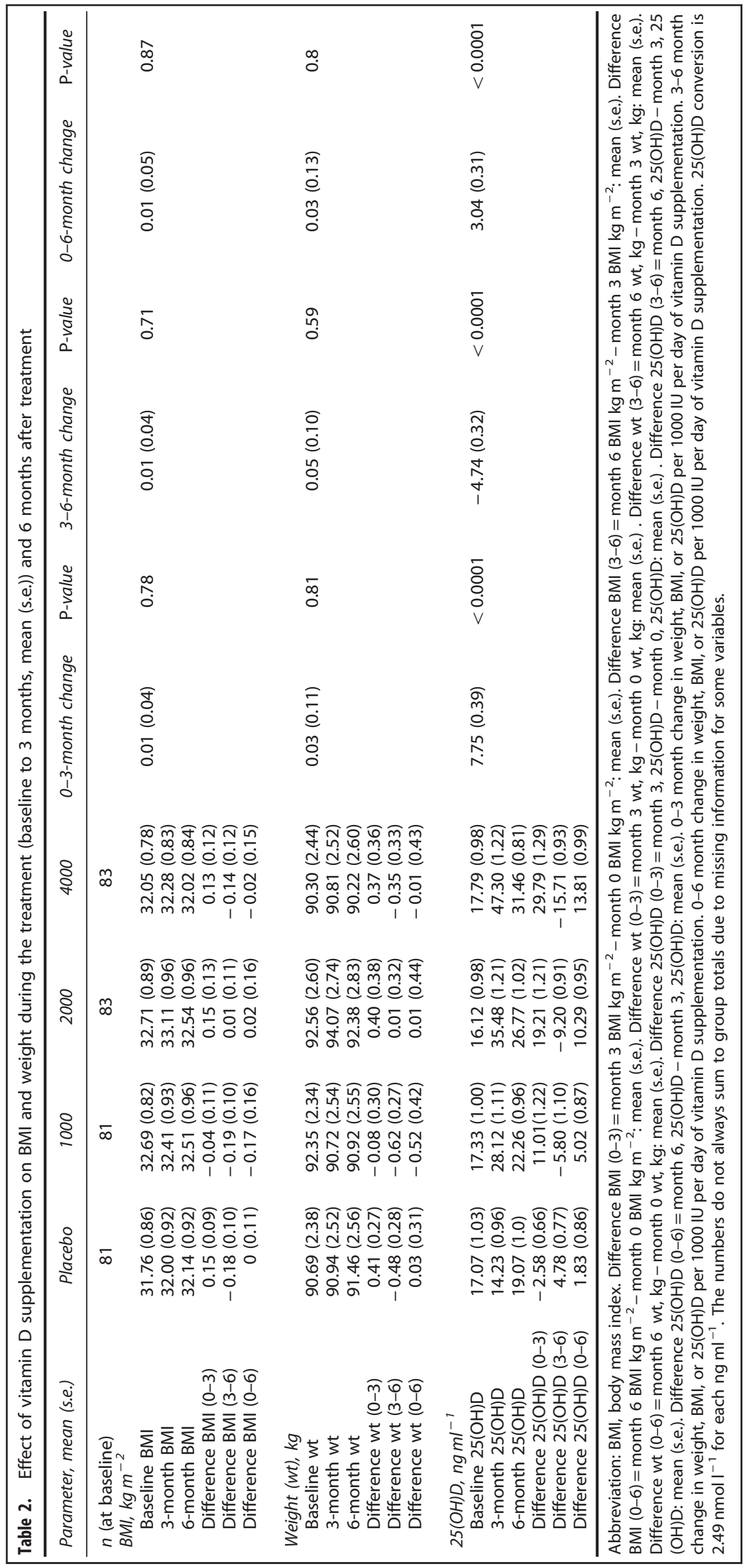

